\title{
Use of Mobile Phone App Interventions to Promote Weight Loss: Meta-Analysis
}

Md Mohaimenul Islam ${ }^{1,2,3}$, MSc; Tahmina Nasrin Poly ${ }^{1,2,3}$, MSc; Bruno Andres Walther ${ }^{4}$, PhD; Yu-Chuan (Jack) $\mathrm{Li}^{1,2,3,5}, \mathrm{PhD}$

${ }^{1}$ Graduate Institute of Biomedical Informatics, College of Medical Science and Technology, Taipei Medical University, Taipei, Taiwan

${ }^{2}$ International Center for Health Information Technology (ICHIT), Taipei Medical University, Taipei, Taiwan

${ }^{3}$ Research Center of Big Data and Meta-Analysis, Wan Fang Hospital, Taipei Medical University, Taipei, Taiwan

${ }^{4}$ Department of Biological Sciences, National Sun Yat-Sen University, Kaohsiung, Taiwan

${ }^{5}$ Department of Dermatology, Wan Fang Hospital, Taipei Medical University, Taipei, Taiwan

\section{Corresponding Author:}

Yu-Chuan (Jack) Li, PhD

Graduate Institute of Biomedical Informatics

College of Medical Science and Technology

Taipei Medical University

Taipei

Taiwan

Phone: 88688622736166

Email: jaak88@gmail.com

\section{Abstract}

Background: Obesity and lack of physical activity are major health risk factors for many life-threatening diseases, such as cardiovascular diseases, type 2 diabetes, and cancer. The use of mobile app interventions to promote weight loss and boost physical activity among children and adults is fascinating owing to the demand for cutting-edge and more efficient interventions. Previously published studies have examined different types of technology-based interventions and their impact on weight loss and increase in physical activity, but evidence regarding the impact of only a mobile phone app on weight loss and increase in physical activity is still lacking.

Objective: The main objective of this study was to assess the efficacy of a mobile phone app intervention for reducing body weight and increasing physical activity among children and adults.

Methods: PubMed, Google Scholar, Scopus, EMBASE, and the Web of Science electronic databases were searched for studies published between January 1, 2000, and April 30, 2019, without language restrictions. Two experts independently screened all the titles and abstracts to find the most appropriate studies. To be included, studies had to be either a randomized controlled trial or a case-control study that assessed a mobile phone app intervention with body weight loss and physical activity outcomes. The Cochrane Collaboration Risk of Bias tool was used to examine the risk of publication bias.

Results: A total of 12 studies involving a mobile phone app intervention were included in this meta-analysis. Compared with the control group, the use of a mobile phone app was associated with significant changes in body weight $(-1.07 \mathrm{~kg}, 95 \% \mathrm{CI}-1.92$ to $-0.21, P=.01)$ and body mass index $(-0.45 \mathrm{~kg} / \mathrm{m} 2,95 \% \mathrm{CI}-0.78$ to $-0.12, P=.008)$. Moreover, a nonsignificant increase in physical activity was observed $(0.17,95 \% \mathrm{CI}-2.21$ to $2.55, P=.88)$.

Conclusions: The findings of this study demonstrate the promising and emerging efficacy of using mobile phone app interventions for weight loss. Future studies are needed to explore the long-term efficacy of mobile app interventions in larger samples.

(JMIR Mhealth Uhealth 2020;8(7):e17039) doi: $\underline{10.2196 / 17039}$

\section{KEYWORDS}

mobile app; mHealth; obesity; physical activity; weight gain prevention 


\section{Introduction}

Overweight (BMI $\geq 25 \mathrm{~kg} / \mathrm{m}^{2}$ ), obesity (BMI $\geq 30 \mathrm{~kg} / \mathrm{m}^{2}$ ), and physical inactivity are major preventable public health problems that are linked to increased risks of chronic diseases such as diabetes, high blood pressure, heart disease, and cancer [1]. In 2016, over 1.9 billion (39\%) and over 650 million (13\%) adults older than 18 years were overweight and obese, respectively, and the prevalence of overweight and obesity has nearly tripled since 1980 [2]. Reducing weight and improving physical activity are thus important priorities to minimize the burden associated with overweight and obesity-related comorbidities. A high number of studies have already demonstrated that a change in lifestyle can help reduce and maintain weight $[3,4]$. However, for many people, it is very difficult to change their lifestyle and maintain weight loss [5]. Therefore, an intervention that helps to motivate people to undertake a change in lifestyle, offers pragmatic goal settings, and offers feedback on activity rates can help maintain weight loss and greatly increase physical activity [6,7].

The mobile phone has become a very important medium of communication throughout the world [8], and approximately $75 \%$ of adults have used different kinds of mobile interventions $[9,10]$. Therefore, the use of a mobile phone intervention could be a promising approach for disease management and prevention that has a huge potential to reach out to the vast majority of the population [11]. Researchers have been using mobile phone interventions to support behavioral change by providing more interactive and timely access to relevant information and delivering context-specific prompt assistance [12]. Recently, the use of mobile apps has led to notable success in obesity control, weight reduction, physical activity increase, and quality of life improvement [13]. App-based interventions have already been shown to be cost-effective and to reduce the immense barriers associated with more traditional approaches. However, the effectiveness and success of a mobile app intervention also relies on how the intervention has been developed. A mobile app intervention delivery system has the capacity to reach each participant effectively and efficiently, and it is thus an innovative way to manage weight and increase physical activity.

The use of a mobile app is a rapidly expanding area of research for disease management and behavioral change. Therefore, the purpose of this systematic review and meta-analysis was to evaluate the current evidence for the feasibility of a mobile phone intervention. Our study updates and extends the scope of a prior systematic review and meta-analysis in three ways [14]. First, we included two more randomized controlled trials than the previous review. Second, we provided more subgroup analyses than the previous review. Third, we extended the sample size and characteristics, such as age, gender, geographic region, and features of the intervention, to evaluate mobile app efficacy for weight loss and increased physical activity.

\section{Methods}

\section{Guidelines}

This systematic review was conducted in accordance with the Meta-Analysis of Observational Studies in Epidemiology guidelines [15] and the Preferred Reporting Items for Systematic Reviews and Meta-Analyses standard (Multimedia Appendix 1) $[16]$.

\section{Literature Search}

PubMed, Google Scholar, Scopus, EMBASE, and the Web of Science electronic databases were searched for studies published between January 1, 2000, and April 30, 2019, without language restrictions. The search was conducted by two experts (MMI and TNP) using combinations of relevant search terms and Boolean operators as follows: "mobile apps" AND "weight loss" OR "weight control" OR "obesity" OR "BMI” OR "body mass index" (Multimedia Appendix 2). There was no language or data restriction for the initial search. However, we did not consider any gray literature for unpublished studies (abstracts and conference proceedings) in the initial search. Such unpublished studies were not considered because they did not pass a proper peer-review process. We then used EndNote X7 (Thomson Reuters) to remove any duplicate publication.

\section{Other Resources Search}

We carefully checked all the retrieved systematic reviews and meta-analyses in order to find further relevant studies.

\section{Eligibility Criteria}

Two experts (MMI and TNP) independently screened all the titles and abstracts to find the most appropriate full-text studies for inclusion. They then further screened all full-text studies for quantitative synthesis of evidence and recorded the inclusion and exclusion criteria. Any disagreements over the inclusion and exclusion criteria that arose during this stage were subsequently resolved by the main investigator (YCL) of this study. We considered all studies if they met the following criteria: (1) published in English; (2) reported a mobile app intervention for change in body weight, BMI, or waist circumference; and (3) reported a mobile app for weight loss among children and adults compared with a control group.

Studies were excluded if they met the following criteria: (1) review or methodology study, short communication, or letter to the editor; (2) case report or case series; (3) no control group; (4) outcomes of interest were other diseases except for a diagnosis of obesity; and (5) other types of mobile phone interventions like text messaging.

\section{Data Extraction}

A predefined standard procedure was used to retrieve all the information from the included studies by the same two authors (MMI and TNP). They also used the Review Manager software (RevMan-5) to check the accuracy of the included studies. They collected the following information from all the included studies: (1) methods: number of studies, number of patients, age of participants, gender of participants or patients, study period, inclusion and exclusion criteria, and intervention and follow-up 
duration; (2) results: study characteristics, target group outcome, intervention characteristics, type of intervention, change of participant's behavior, mean changes from baseline, variation that was reported as SD or $95 \% \mathrm{CI}$, and bias assessment; and (3) discussion: main findings, suggestions, intended recommendations, and limitations.

\section{Study Quality Assessment}

The methodological quality of the included observational design studies was evaluated in accordance with the Cochrane Handbook for Systematic Reviews of Intervention [17]. This guideline is used to evaluate how well-randomized controlled trials were conducted to avoid bias based on a total of seven criteria. The following elements are reported in these guidelines: random sequence generation, allocation concealment, blinding of participants and personnel, blinding of outcome assessment, incomplete outcome data, selective reporting, and other bias. Therefore, the total score was used to assess the quality of the included studies as low, uncertain, or high risk.

\section{Statistical Analysis}

The meta-analysis was performed to evaluate mobile app interventions for weight loss. A random-effect model was used to obtain an overall effect size. We calculated the standardized mean difference between the experimental group (mobile app) and control group using the mean, standard deviation, and total number of individuals. Moreover, an effect size and standard error for outcome (weight loss, BMI, and physical activity) were also calculated. We calculated the effect size with a $95 \% \mathrm{CI}$, and statistical significance was considered at a $P$ value $<.05$. The chi-square $(\mathrm{Q})$ statistic and $\mathrm{I}^{2}$ were also calculated to determine the sources of heterogeneity between the studies. If the $P$ value of the chi-square test was $>.05$, the findings of the study were considered to be due to chance. Homogeneity between the studies was determined if the $P$ value was $>.05$. The $\mathrm{I}^{2}$ value quantitatively determines heterogeneity. The heterogeneity between studies was categorized as very low, low, medium, and high if the $\mathrm{I}^{2}$ values were $0 \%-25 \%, 25 \%-50 \%$, $50 \%-75 \%$, and more than $75 \%$, respectively [18]. A random-effect model was utilized because it is the appropriate model to calculate the effect size if the included studies are heterogeneous, and for homogenous studies, fixed-effect models are appropriate [19]. In addition, a funnel plot was generated to assess publication bias, and it was evaluated by the Egger method. All analyses were conducted using Comprehensive Meta-Analysis software (CMA) Version 2.

\section{Results}

\section{Study Selection}

The literature search of the electronic databases yielded 1523 studies. After our review of the titles and abstracts, a total of 1495 studies were excluded either because of duplication or because of the lack of adherence to our topic. Consequently, only 28 studies underwent full-text review. We also checked their reference lists for further relevant studies and retrieved one additional study. However, 16 more studies were then excluded because they did not meet the inclusion criteria mentioned previously. Finally, a total of 12 studies were included in our meta-analysis [13,20-30]. The flow chart of our systematic literature search is presented in Figure 1. 
Figure 1. Flow chart of the study search and selection.

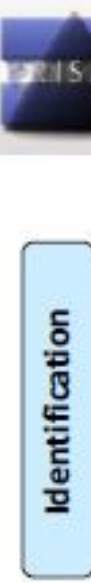

\section{PRISMA 2009 Flow Diagram}
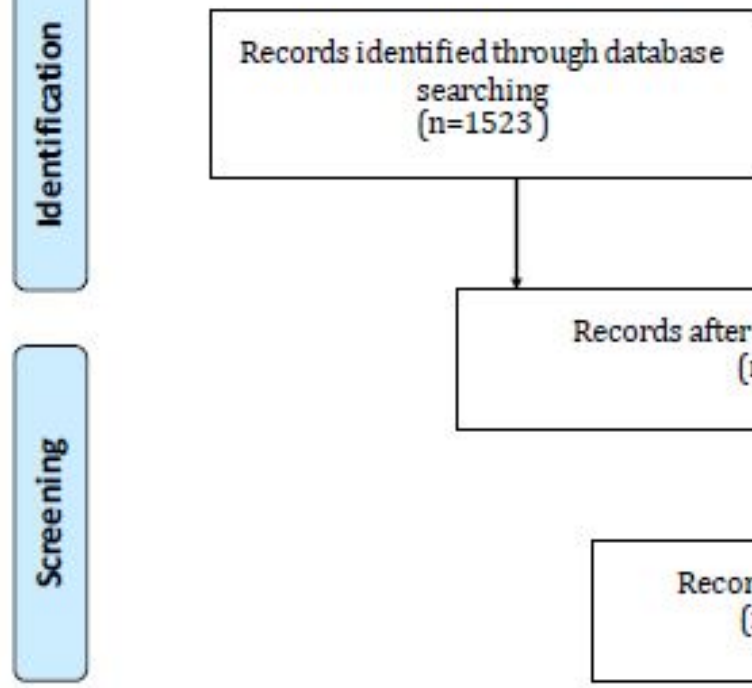
$(\mathrm{n}=1523)$
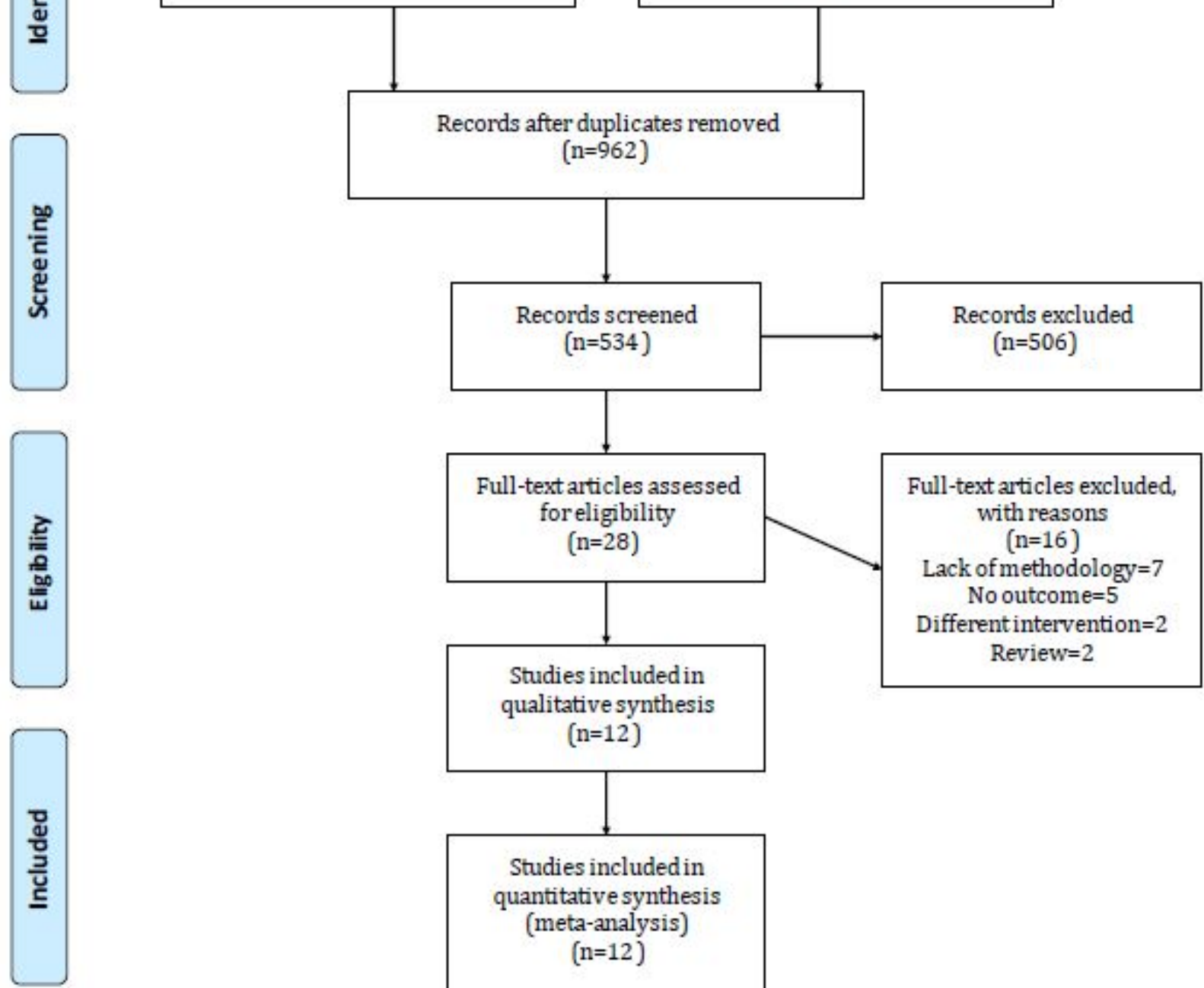

qualitative synthesis $(n=12)$

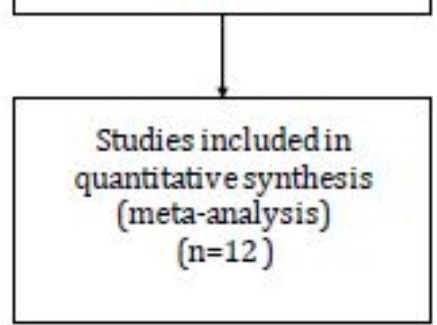

\section{Study Characteristics}

The studies included in our meta-analysis were 11 randomized controlled trials and 1 case-control study (Table 1). The publication year ranged from 2010 to 2019 . The studies included child and adult participants, with a total of 792 experimental participants and 799 controls. Five studies were published in Australia, four in North America, two in Europe, and one in Asia. A total of 1714 participants were included, and the sample size ranged from 35 to 361 . Most of the participants were female. The proportion of female participants ranged from $85 \%$ to $100 \%$, and the mean age of the participants ranged from 12.7 to 44.9 years. The follow-up period of the included studies ranged from 6 weeks to 9 months. Moreover, age, gender, marital status, and education level were used as baseline variables, and mobile apps, email, etc were considered as study interventions (Table 2). 
Table 1. Characteristics of the studies included in the meta-analysis.

\begin{tabular}{|c|c|c|c|c|c|c|c|}
\hline $\begin{array}{l}\text { First author } \\
\text { (year) }\end{array}$ & Country & $\begin{array}{l}\text { Study } \\
\text { design }\end{array}$ & $\begin{array}{l}\text { Study } \\
\text { sam- } \\
\text { ple }\end{array}$ & $\begin{array}{l}\text { Male, } \\
\%\end{array}$ & $\begin{array}{l}\text { Age } \\
\text { (years), } \\
\text { mean }\end{array}$ & $\begin{array}{l}\text { Study du- } \\
\text { ration }\end{array}$ & Inclusion criteria \\
\hline $\begin{array}{l}\text { Patel } \\
\text { (2019) }\end{array}$ & USA & $\mathrm{RCT}^{\mathrm{a}}$ & 100 & 16 & 42.7 & 3 months & $\begin{array}{l}\text { Age } 21-65 \text { years with } \\
\text { BMI } 25-45 \mathrm{~kg} / \mathrm{m}^{2} \text {, and } \\
\text { willingness to reduce } \\
\text { weight through dietary } \\
\text { change. Availability of } \\
\text { an iPhone or Android } \\
\text { smartphone and personal } \\
\text { email address, and ability } \\
\text { to read and write in En- } \\
\text { glish. }\end{array}$ \\
\hline
\end{tabular}

$\begin{array}{lllllll}\begin{array}{l}\text { Farinelli } \\ \text { (2016) }\end{array} & \text { Australia } & \text { RCT } & 258 & 40.7 & 28.1 & 9 \text { months }\end{array}$

(2016)

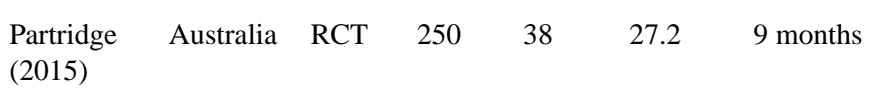

Age 18-35 years, BMI $25.0-31.9 \mathrm{~kg} / \mathrm{m}^{2}$ or $23.0-$ $24.9 \mathrm{~kg} / \mathrm{m}^{2}$ with reported weight gain greater than $2 \mathrm{~kg}$ over the previous 12 months. Fruit intake of less than two servings daily, vegetable intake of less than five servings daily, and $\mathrm{SSB}^{\mathrm{b}}$ intake of at least $1 \mathrm{~L}$ weekly. Energy-dense meals prepared away from home more than once per week. Owning a mobile phone capable of receiving text messages and having access to the internet at least once a week.

$$
\text { (2015) }
$$

BMI $25.0-31.9 \mathrm{~kg} / \mathrm{m}^{2}$ or $23.0-24.9 \mathrm{~kg} / \mathrm{m}^{2}$ with reported weight gain greater than $2 \mathrm{~kg}$ over the previous 12 months, fruit intake of less than two servings daily, vegetable intake of less than five servings daily, SSB intake of at least $1 \mathrm{~L}$ weekly, energy-dense meals prepared away from home more than once per week, etc.

Exclusion criteria

Outcomes

Enrollment in other weight Weight and loss programs, use of MyFit- BMI nessPal to track diet in the past 6 months, loss of $\geq 10$ $\mathrm{lb}$, or use of weight loss medication in the past 6 months. Moreover, pregnancy and disorders, such as cancer, eating disorders, uncontrolled hypertension, diabetes mellitus, cardiovascular events, and congestive heart failure.

Pregnancy or plan for preg- Weight, nancy within the next 9 months, enrollment in another mobile app-based weight loss program, weight reduction more than $10 \mathrm{~kg}$ voluntarily in the past 3 months, taking medications that cause more than $2 \mathrm{~kg}$ of weight gain, medical conditions that preclude following dietary or physical recommendations, history of disorders like eating disorders, and inability to read or write in English.

Pregnancy or plan for pregnancy within the study period, enrollment in other mobile app-based weight loss programs, reduction in weight greater than $10 \mathrm{~kg}$ in the past 3 months, use of medications that help to gain weight greater than $2 \mathrm{~kg}$, other medical conditions that preclude following dietary or physical activity recommendations, and inability to speak English.

Current, planned, or previ- Weight ous pregnancy within the last 6 months, hemodialysis, life expectancy less than 6 months, lack of interest in weight loss, or current use of other kinds of apps for weight loss.
Weight, BMI, MPA $^{\mathrm{d}}$, and VPA ${ }^{\mathrm{e}}$
Age $\geq 18$ years, $\mathrm{BMI} \geq 25$ $\mathrm{kg} / \mathrm{m}^{2}$, and smartphone ownership. 


\begin{tabular}{|c|c|c|c|c|c|c|c|c|c|}
\hline $\begin{array}{l}\text { First author } \\
\text { (year) }\end{array}$ & Country & $\begin{array}{l}\text { Study } \\
\text { design }\end{array}$ & $\begin{array}{l}\text { Study } \\
\text { sam- } \\
\text { ple }\end{array}$ & $\begin{array}{l}\text { Male, } \\
\%\end{array}$ & $\begin{array}{l}\text { Age } \\
\text { (years), } \\
\text { mean }\end{array}$ & $\begin{array}{l}\text { Study du- } \\
\text { ration }\end{array}$ & Inclusion criteria & Exclusion criteria & Outcomes \\
\hline $\begin{array}{l}\text { Hebden } \\
(2014)\end{array}$ & Australia & RCT & 41 & 15 & 22.6 & 3 months & $\begin{array}{l}\text { BMI } 24.00-31.99 \mathrm{~kg} / \mathrm{m}^{2} \\
\text { with weight gain greater } \\
\text { than } 2 \mathrm{~kg} \text { in the past } 12 \\
\text { months, age } 18-35 \text { years, } \\
\text { moderate intensity physi- } \\
\text { cal activity }<60 \text { min/day, } \\
\text { SSB intake of at least } 1 \\
\mathrm{~L} \text { weekly, fruit intake of } \\
\text { less than two servings } \\
\text { daily, vegetable intake of } \\
\text { less than five servings } \\
\text { daily, or at least two ener- } \\
\text { gy-dense takeaway meals } \\
\text { weekly. }\end{array}$ & $\begin{array}{l}\text { Inability to receive SMS } \\
\text { messages or no regular inter- } \\
\text { net access, a diet required } \\
\text { for medical reasons, medical } \\
\text { conditions that influence } \\
\text { body weight or ability to } \\
\text { comply with the interven- } \\
\text { tion, intake of medications } \\
\text { or herbal preparations that } \\
\text { might influence body } \\
\text { weight, enrollment in weight } \\
\text { loss programs, pregnancy, } \\
\text { or plan for pregnancy in the } \\
\text { next } 3 \text { months. }\end{array}$ & $\begin{array}{l}\text { Weight, } \\
\text { BMI, and } \\
\text { PA }\end{array}$ \\
\hline $\begin{array}{l}\text { Smith } \\
(2014)\end{array}$ & Australia & $\mathrm{RCT}$ & 361 & 100 & 12.7 & 7 months & $\begin{array}{l}\text { Male students in their } \\
\text { first year at the study } \\
\text { schools completed a } \\
\text { short screening question- } \\
\text { naire. }\end{array}$ & $\mathrm{NR}^{\mathrm{f}}$ & $\begin{array}{l}\text { BMI and waist } \\
\text { circumference }\end{array}$ \\
\hline $\begin{array}{l}\text { Glynn } \\
(2014)\end{array}$ & Ireland & $\mathrm{RCT}$ & 139 & 32 & 44 & 2 months & $\begin{array}{l}\text { Age }>16 \text { years and active } \\
\text { use of an Android smart- } \\
\text { phone. }\end{array}$ & $\begin{array}{l}\text { No android smartphone, } \\
\text { acute psychiatric illness, } \\
\text { pregnancy, or inability to } \\
\text { undertake moderate exer- } \\
\text { cise. }\end{array}$ & $\begin{array}{l}\text { Weight, BMI, } \\
\text { and PA }\end{array}$ \\
\hline $\begin{array}{l}\text { Brindal } \\
(2013)\end{array}$ & Australia & $\mathrm{RCT}$ & 58 & 0 & 42 & 2 months & $\begin{array}{l}\text { BMI }>25 \mathrm{~kg} / \mathrm{m}^{2} \text { and abil- } \\
\text { ity to measure weight at } \\
\text { home. }\end{array}$ & $\begin{array}{l}\text { Medical conditions that are } \\
\text { likely to interfere with the } \\
\text { ability to undertake the meal } \\
\text { replacement program (eg, } \\
\text { pregnancy, breastfeeding, } \\
\text { active cancer, gastrointesti- } \\
\text { nal disorders, and type } 1 \text { dia- } \\
\text { betes). }\end{array}$ & Weight \\
\hline $\begin{array}{l}\text { Carter } \\
(2013)\end{array}$ & UK & RCT & 128 & 23.3 & 41.2 & 6 months & $\begin{array}{l}\mathrm{BMI} \geq 27 \mathrm{~kg} / \mathrm{m}^{2} \text {, age } 18- \\
65 \text { years, and willingness } \\
\text { to commit the necessary } \\
\text { time and effort to the } \\
\text { study. Ability to read and } \\
\text { write in English, ability } \\
\text { to access the internet, and } \\
\text { willingness to be random- } \\
\text { ized to one of three } \\
\text { groups. }\end{array}$ & $\begin{array}{l}\text { Pregnancy, breast feeding, } \\
\text { plan for pregnancy, use of } \\
\text { antiobesity medication or } \\
\text { medication/insulin for dia- } \\
\text { betes, surgery for weight } \\
\text { loss, and use of the antide- } \\
\text { pressant sertraline. }\end{array}$ & $\begin{array}{l}\text { Weight and } \\
\text { BMI }\end{array}$ \\
\hline $\begin{array}{l}\text { Allen } \\
(2013)\end{array}$ & USA & RCT & 35 & 22.1 & 44.9 & 6 months & $\begin{array}{l}\text { Age } 21-65 \text { years, BMI } \\
28-42 \mathrm{~kg} / \mathrm{m}^{2} \text {, ownership } \\
\text { of an iPhone or Android } \\
\text { phone, willingness to } \\
\text { download the app to be } \\
\text { used on their device. }\end{array}$ & $\begin{array}{l}\text { History of myocardial infarc- } \\
\text { tion, angina, coronary artery } \\
\text { bypass graft surgery, percu- } \\
\text { taneous transluminal coro- } \\
\text { nary angioplasty, congestive } \\
\text { heart failure, and diabetes. } \\
\text { Current participation in oth- } \\
\text { er weight loss programs, } \\
\text { pregnancy, plan for pregnan- } \\
\text { cy in the next } 6 \text { months, use } \\
\text { of weight loss medications, } \\
\text { and history of psychiatric } \\
\text { illness, alcohol, or substance } \\
\text { abuse within the past } 12 \\
\text { months. }\end{array}$ & $\begin{array}{l}\text { Weight, BMI, } \\
\text { and waist cir- } \\
\text { cumference }\end{array}$ \\
\hline
\end{tabular}




\begin{tabular}{|c|c|c|c|c|c|c|c|c|c|}
\hline $\begin{array}{l}\text { First author } \\
\text { (year) }\end{array}$ & Country & $\begin{array}{l}\text { Study } \\
\text { design }\end{array}$ & $\begin{array}{l}\text { Study } \\
\text { sam- } \\
\text { ple }\end{array}$ & $\begin{array}{l}\text { Male, } \\
\%\end{array}$ & $\begin{array}{l}\text { Age } \\
\text { (years), } \\
\text { mean }\end{array}$ & $\begin{array}{l}\text { Study du- } \\
\text { ration }\end{array}$ & Inclusion criteria & Exclusion criteria & Outcomes \\
\hline $\begin{array}{l}\text { McGrievy } \\
(2011)\end{array}$ & USA & RCT & 96 & 24.7 & 44 & 6 months & $\begin{array}{l}\text { Age } 18-60 \text { years and } \\
\text { BMI } 25-45 \mathrm{~kg} / \mathrm{m}^{2}\end{array}$ & $\begin{array}{l}\text { Smoking, unstable medical } \\
\text { status, uncontrolled thyroid } \\
\text { condition, inability to attend } \\
\text { the three monitoring visits } \\
\text { or improve the walking sta- } \\
\text { tus, psychiatric illness, alco- } \\
\text { hol consumption, drug de- } \\
\text { pendency, eating disorders, } \\
\text { enrollment in another weight } \\
\text { loss program, pregnancy, } \\
\text { breast feeding, and plan for } \\
\text { pregnancy within the next } 6 \\
\text { months. }\end{array}$ & BMI and PA \\
\hline Li (2010) & $\begin{array}{l}\text { South Ko- } \\
\text { rea }\end{array}$ & $\mathrm{CCS}^{\mathrm{g}}$ & 36 & NR & 28.5 & 6 weeks & $\begin{array}{l}\text { Different ages and blood } \\
\text { groups because of individ- } \\
\text { ual lifestyle and health } \\
\text { effects according to } \\
\text { blood group and the re- } \\
\text { quirement of various } \\
\text { amounts of calories } \\
\text { based on gender. }\end{array}$ & NR & $\begin{array}{l}\text { Weight and } \\
\text { BMI }\end{array}$ \\
\hline
\end{tabular}

${ }^{\mathrm{a}} \mathrm{RCT}$ : randomized controlled trial.

${ }^{\mathrm{b}} \mathrm{SSB}$ : sugar-sweetened beverage.

${ }^{\mathrm{c}} \mathrm{PA}$ : physical activity.

${ }^{\mathrm{d}}$ MPA: moderate physical activity.

${ }^{\mathrm{e}}$ VPA: vigorous physical activity.

${ }^{\mathrm{f}} \mathrm{NR}$ : not reported.

${ }^{\mathrm{g}}$ CCS: case-control study. 
Table 2. Descriptions of baseline, interventions, apps, and findings of the included studies.

\begin{tabular}{|c|c|c|c|c|c|c|c|c|}
\hline $\begin{array}{l}\text { First author } \\
\text { (year) }\end{array}$ & $\begin{array}{l}\text { Baseline vari- } \\
\text { ables }\end{array}$ & $\begin{array}{l}\text { Intervention } \\
\text { type }\end{array}$ & $\begin{array}{l}\text { App descrip- } \\
\text { tion }\end{array}$ & $\begin{array}{l}\text { Control group } \\
\text { treatment }\end{array}$ & $\begin{array}{l}\text { Difference of } \\
\text { the interven- } \\
\text { tion group, } \\
\text { mean (SD) }\end{array}$ & $\begin{array}{l}\text { Difference } \\
\text { of the con- } \\
\text { trol group, } \\
\text { mean (SD) }\end{array}$ & Inference & $\begin{array}{l}\text { Recommenda- } \\
\text { tion }\end{array}$ \\
\hline $\begin{array}{l}\text { Patel } \\
(2019)\end{array}$ & $\begin{array}{l}\text { Age, gender, } \\
\text { marital status, } \\
\text { race/ethnicity, } \\
\text { education, em- } \\
\text { ployment sta- } \\
\text { tus, annual } \\
\text { household in- } \\
\text { come, body } \\
\text { mass index } \\
\text { category, self- } \\
\text { monitoring of } \\
\text { diet frequen- } \\
\text { cy, and type of } \\
\text { smartphone }\end{array}$ & $\begin{array}{l}\text { App, email, } \\
\text { MyFitnessPal, } \\
\text { mobile, and in- } \\
\text { ternet }\end{array}$ & $\begin{array}{l}\text { Weight loss } \\
\text { goal, calorie } \\
\text { goal, self- } \\
\text { monitoring of } \\
\text { body weight, } \\
\text { dietary intake, } \\
\text { rea-time feed- } \\
\text { back, skill } \\
\text { training, and } \\
\text { reminder of } \\
\text { the goal }\end{array}$ & $\begin{array}{l}\text { Self-regulation, } \\
\text { email, and action } \\
\text { plans via weekly } \\
\text { email }\end{array}$ & $-1.8(1.53)$ & $-2.55(1.11)$ & $\begin{array}{l}\text { The mobile } \\
\text { app is an effec- } \\
\text { tive interven- } \\
\text { tion for clini- } \\
\text { cally meaning- } \\
\text { ful weight } \\
\text { loss. }\end{array}$ & $\begin{array}{l}\text { Stand-alone } \\
\text { digital health } \\
\text { treatments may } \\
\text { be a viable op- } \\
\text { tion for those } \\
\text { looking for a } \\
\text { lower intensity } \\
\text { approach. }\end{array}$ \\
\hline $\begin{array}{l}\text { Farinelli } \\
(2016)\end{array}$ & 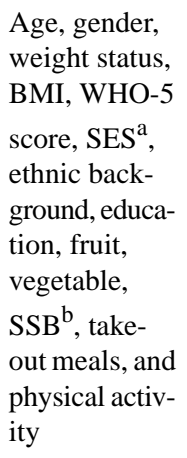 & $\begin{array}{l}\text { Mobile app, } \\
\text { email, online } \\
\text { weight tracker, } \\
\text { physical activi- } \\
\text { ty planner, a } \\
\text { blog facility for } \\
\text { communication, } \\
\text { and printable } \\
\text { eating chart }\end{array}$ & $\begin{array}{l}\text { Smart mobile } \\
\text { apps for educa- } \\
\text { tion and self- } \\
\text { monitoring }\end{array}$ & $\begin{array}{l}\text { Four text mes- } \\
\text { sages, one on } \\
\text { each key behav- } \\
\text { ior, and a two- } \\
\text { page handout } \\
\text { based on dietary } \\
\text { guidelines. }\end{array}$ & $-3.8(4.9)$ & $-0.80(3.7)$ & $\begin{array}{l}\text { The mHealth } \\
\text { intervention } \\
\text { has the poten- } \\
\text { tial to reduce } \\
\text { weight and } \\
\text { improve physi- } \\
\text { cal activity. }\end{array}$ & $\begin{array}{l}\text { Replication of } \\
\text { trials and } \\
\text { widespread } \\
\text { adoption of this } \\
\text { model are need- } \\
\text { ed. }\end{array}$ \\
\hline $\begin{array}{l}\text { Laing } \\
(2014)\end{array}$ & $\begin{array}{l}\text { Gender, self- } \\
\text { reported race, } \\
\text { education, an- } \\
\text { nual income, } \\
\text { and type of } \\
\text { smartphone }\end{array}$ & $\begin{array}{l}\text { App and usual } \\
\text { care plan }\end{array}$ & $\begin{array}{l}\text { MyFitnessPal } \\
\text { app }\end{array}$ & $\begin{array}{l}\text { Counseling and } \\
\text { one-page educa- } \\
\text { tional handout for } \\
\text { eating plan }\end{array}$ & $-0.03(4.64)$ & $0.27(4.64)$ & $\begin{array}{l}\text { The app was } \\
\text { an effective } \\
\text { tool for reduc- } \\
\text { ing weight. }\end{array}$ & $\mathrm{NR}^{\mathrm{c}}$ \\
\hline $\begin{array}{l}\text { Hebden } \\
(2014)\end{array}$ & $\begin{array}{l}\text { Age, gender, } \\
\text { SES, educa- } \\
\text { tion, work his- } \\
\text { tory, lives } \\
\text { with parents, } \\
\text { and English } \\
\text { proficiency }\end{array}$ & $\begin{array}{l}\text { App, text mes- } \\
\text { saging, email, } \\
\text { internet forum, } \\
\text { and usual care }\end{array}$ & $\begin{array}{l}\text { Four types of } \\
\text { behavior plans }\end{array}$ & $\begin{array}{l}\text { 10-page printed } \\
\text { book }\end{array}$ & $-1.6(3)$ & $-1.4(3.18)$ & $\begin{array}{l}\text { The app pro- } \\
\text { vided short- } \\
\text { term positive } \\
\text { changes in } \\
\text { weight, nutri- } \\
\text { tion, and phys- } \\
\text { ical activity. }\end{array}$ & $\begin{array}{l}\text { More studies } \\
\text { are needed to } \\
\text { explore engage- } \\
\text { ment and per- } \\
\text { sonalized sup- } \\
\text { port }\end{array}$ \\
\hline
\end{tabular}




\begin{tabular}{|c|c|c|c|c|c|c|c|c|}
\hline $\begin{array}{l}\text { First author } \\
\text { (year) }\end{array}$ & $\begin{array}{l}\text { Baseline vari- } \\
\text { ables }\end{array}$ & $\begin{array}{l}\text { Intervention } \\
\text { type }\end{array}$ & $\begin{array}{l}\text { App descrip- } \\
\text { tion }\end{array}$ & $\begin{array}{l}\text { Control group } \\
\text { treatment }\end{array}$ & $\begin{array}{l}\text { Difference of } \\
\text { the interven- } \\
\text { tion group, } \\
\text { mean (SD) }\end{array}$ & $\begin{array}{l}\text { Difference } \\
\text { of the con- } \\
\text { trol group, } \\
\text { mean (SD) }\end{array}$ & Inference & $\begin{array}{l}\text { Recommenda- } \\
\text { tion }\end{array}$ \\
\hline $\begin{array}{l}\text { Smith } \\
(2014)\end{array}$ & $\begin{array}{l}\text { Age, English } \\
\text { language, cul- } \\
\text { tural back- } \\
\text { ground, socioe- } \\
\text { conomic posi- } \\
\text { tion, weight, } \\
\text { height, BMI, } \\
\text { weight status, } \\
\text { and waist cir- } \\
\text { cumference }\end{array}$ & $\begin{array}{l}\text { App, parent } \\
\text { newsletters, } \\
\text { seminars, spot } \\
\text { sessions, } \\
\text { lunchtime phys- } \\
\text { ical activity } \\
\text { monitoring, and } \\
\text { teaching materi- } \\
\text { al }\end{array}$ & $\begin{array}{l}\text { Fitness chal- } \\
\text { lenges, activi- } \\
\text { ty monitoring, } \\
\text { and motiva- } \\
\text { tional mes- } \\
\text { sages }\end{array}$ & $\begin{array}{l}\text { Traditional ap- } \\
\text { proaches }\end{array}$ & $0.6(1.21)$ & $0.61(1.07)$ & $\begin{array}{l}\text { The app-based } \\
\text { intervention } \\
\text { helped to im- } \\
\text { prove fitness, } \\
\text { movement } \\
\text { skill, and key } \\
\text { weight-related } \\
\text { behavior. }\end{array}$ & $\begin{array}{l}\text { More studies re- } \\
\text { quire to capture } \\
\text { objective data } \\
\text { on app usage } \\
\text { throughout the } \\
\text { intervention pe- } \\
\text { riod and find } \\
\text { out the associa- } \\
\text { tion. It is also } \\
\text { important to } \\
\text { add some fea- } \\
\text { tures like gamifi- } \\
\text { cation. }\end{array}$ \\
\hline $\begin{array}{l}\text { Glynn } \\
(2014)\end{array}$ & $\begin{array}{l}\text { Gender, age, } \\
\text { systolic and } \\
\text { diastolic blood } \\
\text { pressure, } \\
\text { weight, BMI, } \\
\text { HADS }{ }^{\text {d }, ~ E Q-~} \\
\text { VAS }^{\mathrm{e}}, \mathrm{EQ}- \\
\text { 5D } \text {, and daily } \\
\text { step count }\end{array}$ & $\begin{array}{l}\text { App and usual } \\
\text { care plan }\end{array}$ & $\begin{array}{l}\text { Accupedo-Pro } \\
\text { Pedometer } \\
\text { app }\end{array}$ & $\begin{array}{l}\text { Education pro- } \\
\text { gram about the } \\
\text { benefits of physi- } \\
\text { cal activity and } \\
\text { exercise }\end{array}$ & $-2.2(3.4)$ & $-1.5(4.3)$ & $\begin{array}{l}\text { The mobile } \\
\text { app-based in- } \\
\text { tervention had } \\
\text { a positive im- } \\
\text { pact on weight } \\
\text { loss }\end{array}$ & NR \\
\hline $\begin{array}{l}\text { Brindal } \\
(2013)\end{array}$ & $\begin{array}{l}\text { Weight and di- } \\
\text { etary status }\end{array}$ & $\begin{array}{l}\text { App and } \\
\text { celebrity slim } \\
\text { program }\end{array}$ & $\begin{array}{l}\text { Support apps } \\
\text { like my meals, } \\
\text { my weight, } \\
\text { and my task }\end{array}$ & $\begin{array}{l}\text { Only celebrity } \\
\text { slim program }\end{array}$ & $-2.9(6.4)$ & $-2.1(1)$ & $\begin{array}{l}\text { The app inter- } \\
\text { vention was } \\
\text { useful for } \\
\text { weight loss } \\
\text { and psycholog- } \\
\text { ical changes. }\end{array}$ & $\begin{array}{l}\text { Integrating } \\
\text { more dynamic } \\
\text { stage-based tai- } \\
\text { loring, as } \\
\text { behavioral } \\
\text { changes of indi- } \\
\text { viduals may } \\
\text { further enhance } \\
\text { similar apps in } \\
\text { the future. }\end{array}$ \\
\hline $\begin{array}{l}\text { Carter } \\
(2013)\end{array}$ & $\begin{array}{l}\text { Age, weight, } \\
\text { BMI, body fat, } \\
\text { gender, race, } \\
\text { smoking sta- } \\
\text { tus, occupa- } \\
\text { tion, and edu- } \\
\text { cation }\end{array}$ & App & $\begin{array}{l}\text { Self-monitor- } \\
\text { ing }\end{array}$ & $\begin{array}{l}\text { Food diary and a } \\
\text { calorie-counting } \\
\text { book }\end{array}$ & $-4.6(5.2)$ & $-2.9(5.85)$ & $\begin{array}{l}\text { The mobile } \\
\text { app was an ac- } \\
\text { ceptable and } \\
\text { feasible } \\
\text { weight loss in- } \\
\text { tervention }\end{array}$ & $\begin{array}{l}\text { More studies } \\
\text { are needed to } \\
\text { investigate the } \\
\text { cost of imple- } \\
\text { menting a } \\
\text { smartphone app } \\
\text { intervention } \\
\text { compared with } \\
\text { other types of } \\
\text { interventions }\end{array}$ \\
\hline $\begin{array}{l}\text { Allen } \\
(2013)\end{array}$ & $\begin{array}{l}\text { Age, weight, } \\
\text { BMI, waist } \\
\text { circumfer- } \\
\text { ence, educa- } \\
\text { tion, and mari- } \\
\text { tal status }\end{array}$ & $\begin{array}{l}\text { App and inten- } \\
\text { sive counseling }\end{array}$ & Lose it! & $\begin{array}{l}\text { Comprehensive } \\
\text { counseling }\end{array}$ & $-5.4(4)$ & $-2.5(4.1)$ & $\begin{array}{l}\text { The app inter- } \\
\text { vention had a } \\
\text { positive im- } \\
\text { pact on weight } \\
\text { loss and con- } \\
\text { tributed to be- } \\
\text { havioral } \\
\text { changes. }\end{array}$ & $\begin{array}{l}\text { Need to conduct } \\
\text { a large-scale } \\
\text { population- } \\
\text { based study. }\end{array}$ \\
\hline $\begin{array}{l}\text { McGrievy } \\
(2011)\end{array}$ & NR & $\begin{array}{l}\text { App + podcast } \\
+ \text { twitter }\end{array}$ & $\begin{array}{l}\text { Diet plan and } \\
\text { physical activ- } \\
\text { ity monitoring }\end{array}$ & Podcast only & $-2.57(2.6)$ & $-2.45(4.39)$ & NR & NR \\
\hline
\end{tabular}




\begin{tabular}{|c|c|c|c|c|c|c|c|c|}
\hline $\begin{array}{l}\text { First author } \\
\text { (year) }\end{array}$ & $\begin{array}{l}\text { Baseline vari- } \\
\text { ables }\end{array}$ & $\begin{array}{l}\text { Intervention } \\
\text { type }\end{array}$ & $\begin{array}{l}\text { App descrip- } \\
\text { tion }\end{array}$ & $\begin{array}{l}\text { Control group } \\
\text { treatment }\end{array}$ & $\begin{array}{l}\text { Difference of } \\
\text { the interven- } \\
\text { tion group, } \\
\text { mean (SD) }\end{array}$ & $\begin{array}{l}\text { Difference } \\
\text { of the con- } \\
\text { trol group, } \\
\text { mean (SD) }\end{array}$ & Inference & $\begin{array}{l}\text { Recommenda- } \\
\text { tion }\end{array}$ \\
\hline $\mathrm{Li}(2010)$ & $\begin{array}{l}\text { Age, occupa- } \\
\text { tion, educa- } \\
\text { tion, monthly } \\
\text { income, smok- } \\
\text { ing, drinking, } \\
\text { and exercise } \\
\text { history }\end{array}$ & $\begin{array}{l}\text { Mobile app and } \\
\text { usual care }\end{array}$ & $\begin{array}{l}\text { Mobile apps } \\
\text { that provided } \\
\text { a personal diet } \\
\text { profile based } \\
\text { on gender and } \\
\text { promoted } \\
\text { knowledge } \\
\text { about nutrition } \\
\text { and physical } \\
\text { activity }\end{array}$ & NR & $-1.9(2.3)$ & $-0.9(4.64)$ & $\begin{array}{l}\text { Improved user } \\
\text { satisfaction. }\end{array}$ & $\begin{array}{l}\text { A more effec- } \\
\text { tive study to } \\
\text { motivate partici- } \\
\text { pants and ex- } \\
\text { tend study dura- } \\
\text { tion is required. }\end{array}$ \\
\hline
\end{tabular}

\footnotetext{
${ }^{\mathrm{a}} \mathrm{SES}$ : socioeconomic status.

${ }^{\mathrm{b}} \mathrm{SSB}$ : sugar-sweetened beverage.

${ }^{\mathrm{c}} \mathrm{NR}$ : not reported.

${ }^{\mathrm{d}}$ HADS: Hospital Anxiety and Depression Scale.

${ }^{\mathrm{e}} \mathrm{EQ}-\mathrm{VAS}$ : EuroQol visual analogue scale.

${ }^{f}$ EQ-5D: EuroQol five-dimension scale.
}

\section{Assessment of the Risk of Bias}

Owing to the nature of mobile app interventions, participant blinding is not always feasible in trials. All the 12 studies reported random sequence generation that showed a low risk of bias. Overall, 10 out of the 12 studies were considered to constitute high-quality evidence. Only three studies had blinding of the outcome assessment, and two studies had blinding of the participants and personnel. A summary of the evaluation of the included studies is shown in Figure 2.

Figure 2. Risk bias assessment of the included studies.

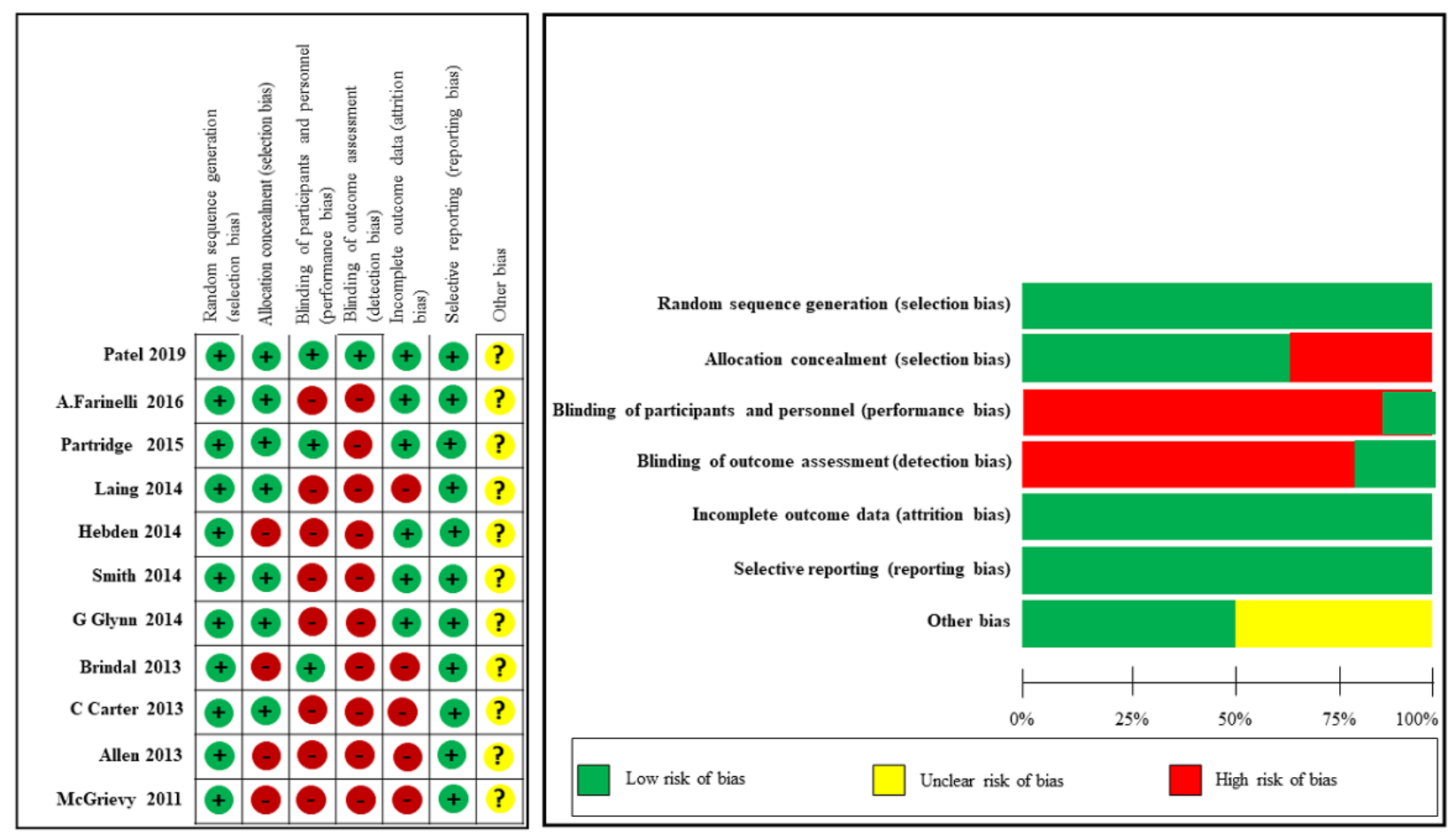

\section{Mobile App Intervention and Weight Loss}

Eleven studies assessed the effectiveness of mobile phone app interventions for reducing body weight. Participants in the intervention group showed a decrease in their body weight
$(-1.07 \mathrm{~kg}, 95 \% \mathrm{CI}-1.92$ to -0.21$)$ when compared with the control group (Figure 3). However, moderate heterogeneity was observed among the studies (heterogeneity $\mathrm{I}^{2}=71.55 \%, \mathrm{Q}=42.65$, $\left.P=.01, \tau^{2}=1.40\right)$. 
Figure 3. Forest plot of mobile phone app interventions and weight loss.

\begin{tabular}{|c|c|c|c|c|c|c|c|}
\hline \multirow[t]{2}{*}{ Studyname } & \multicolumn{7}{|c|}{ Statistics for eadh study } \\
\hline & $\begin{array}{l}\text { Dfference } \\
\text { in means }\end{array}$ & $\begin{array}{c}\text { Standard } \\
\text { error }\end{array}$ & Variance & $\begin{array}{l}\text { Lower } \\
\text { limit }\end{array}$ & $\begin{array}{r}\text { Upper } \\
\text { limit }\end{array}$ & $Z$ value & $P$ value \\
\hline Patel 2019 & 0.370 & 0.357 & 0.127 & -0.329 & 1.069 & 1.037 & .30 \\
\hline Fainelli 2016 & -3.000 & 0.551 & 0.303 & -4.080 & -1.920 & -5.447 & .00 \\
\hline Patridge 2015 & -2.100 & 0.369 & 0.136 & -2.823 & -1.377 & -5.693 & .00 \\
\hline Laing 2014 & -0.300 & 0.637 & 0.406 & -1.549 & 0.949 & -0.471 & .63 \\
\hline Hebden 2014 & -0.200 & 0.865 & 0.749 & -1.896 & 1.496 & -0.231 & .81 \\
\hline Glynn 2014 & -0.700 & 0.817 & 0.668 & -2.302 & 0.902 & -0.857 & 39 \\
\hline Bindal 2013 & -0.800 & 1.183 & 1.399 & -3.119 & 1.519 & -0.676 & .49 \\
\hline Canter 2013 & -1.700 & 1.194 & 1.425 & -4.039 & 0.639 & -1.424 & .15 \\
\hline Allen 2013 & -2.900 & 1.393 & 1.940 & -5.630 & -0.170 & -2.082 & .03 \\
\hline MGGiery 2011 & -0.120 & 0.740 & 0.548 & -1.571 & 1.331 & -0.162 & .87 \\
\hline Lee 2010 & -1.000 & 1.201 & 1.441 & -3.353 & 1.353 & -0.833 & .40 \\
\hline Random effect: & -1.073 & 0.436 & 0.190 & -1.928 & -0.218 & -2.459 & .01 \\
\hline
\end{tabular}

\section{Mobile App Intervention and BMI}

A total of 10 studies evaluated the efficacy of mobile apps for $\mathrm{BMI}$ reduction. The overall pooled findings showed a significant

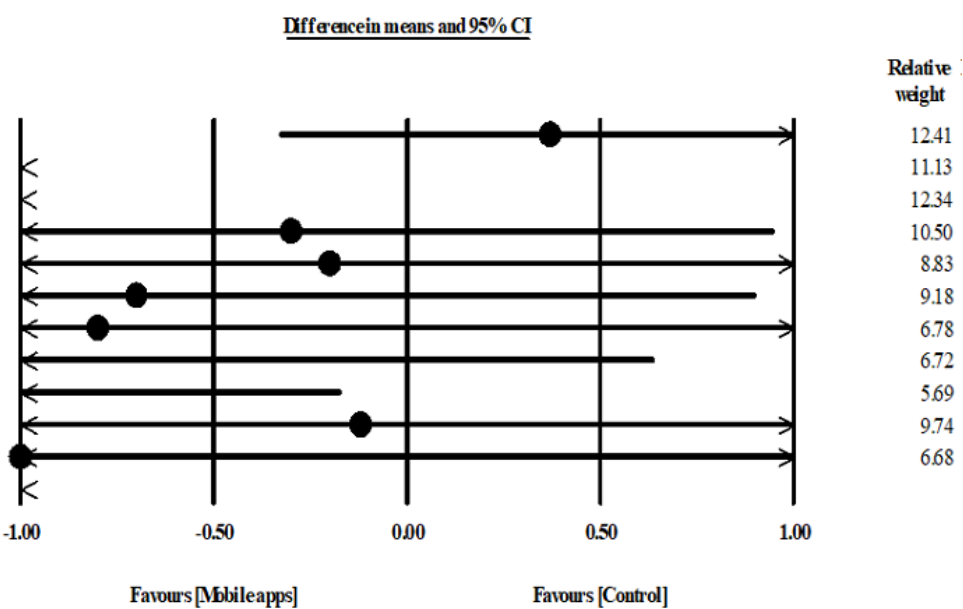

difference in BMI between participants in the mobile phone app intervention group and control group $\left(-0.45 \mathrm{~kg} / \mathrm{m}^{2}, 95 \%\right.$ CI -0.78 to $-0.12, P=.008$, heterogeneity $\mathrm{I}^{2}=77.95 \%, \mathrm{Q}=40.81$, $\left.\tau^{2}=0.17\right)($ Figure 4).

Figure 4. Forest plot of mobile phone app interventions and change in BMI.

\begin{tabular}{|c|c|c|c|c|c|c|c|}
\hline \multirow[t]{2}{*}{ Study name } & \multicolumn{7}{|c|}{ Statistics for ench study } \\
\hline & $\begin{array}{l}\text { Diff erence } \\
\text { in menns }\end{array}$ & $\begin{array}{c}\text { Standard } \\
\text { error }\end{array}$ & Variance & $\begin{array}{l}\text { Lower } \\
\text { limit }\end{array}$ & $\begin{array}{l}\text { Upper } \\
\text { limit }\end{array}$ & $Z$ value & $P$ value \\
\hline Patel 2019 & 0.140 & 0.126 & 0.016 & -0.107 & 0.387 & 1.109 & 26 \\
\hline Farinelli 2016 & -1.040 & 0.191 & 0.036 & -1.414 & -0.666 & -5.448 & .00 \\
\hline Partridge 2015 & -0.600 & 0.156 & 0.024 & -0905 & -0.295 & -3.855 & .00 \\
\hline Hebden 2014 & -0.100 & 0.458 & 0.210 & -0997 & 0.797 & -0218 & 82 \\
\hline Smith 2014 & -0.010 & 0.120 & 0.014 & -0246 & 0.226 & -0.083 & 93. \\
\hline Glynn 2014 & -0.600 & 0.413 & 0.171 & -1.410 & 0.210 & -1.452 & .14 \\
\hline Carter 2013 & -0.600 & 0.732 & 0.536 & -2.035 & 0.835 & -0.820 & 41. \\
\hline Allen 2013 & -1.000 & 0.465 & 0.216 & -1912 & -0.088 & -2.149 & .03 \\
\hline MrGrievy 2011 & -0.650 & 0.701 & 0.491 & -2.024 & 0.724 & -0927 & 35 \\
\hline Lee 2010 & -0.600 & 0316 & 0.100 & -1220 & 0.020 & -1.898 & .05 \\
\hline Random effect: & $: \quad-0.454$ & 0.170 & 0.029 & -0.787 & -0.121 & -2.670 & .00 \\
\hline
\end{tabular}

\section{Mobile App Intervention and Physical Activity}

Seven studies assessed the effectiveness of mobile phone apps for increasing physical activity. The usability and effectiveness of mobile apps had promising but insignificant results (mean

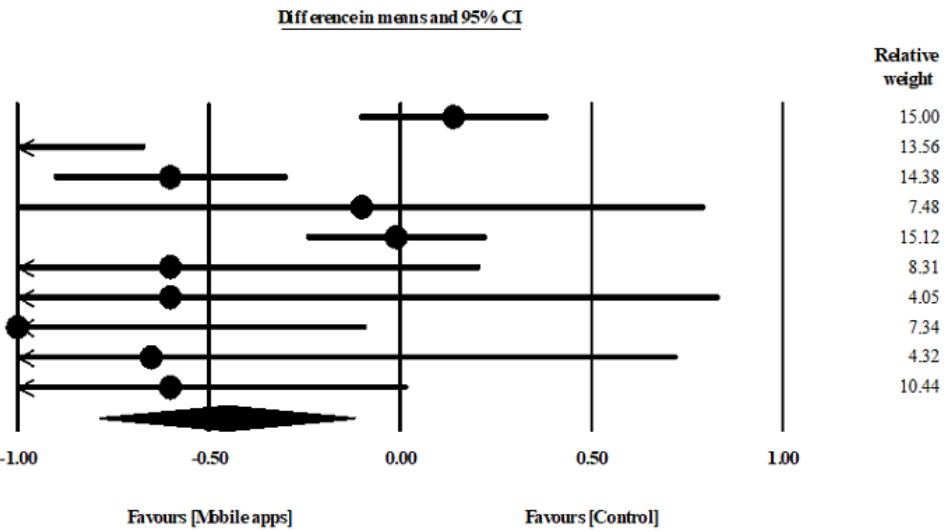

difference $0.17,95 \% \mathrm{CI}-2.21$ to $2.55, P=.88$ ). However, moderate heterogeneity was observed among the included studies (heterogeneity $\mathrm{I}^{2}=74.05 \%, \mathrm{Q}=23.12$, and $\tau^{2}=3.45$ ) (Figure 5). 
Figure 5. Forest plot of mobile phone apps for increased physical activity.

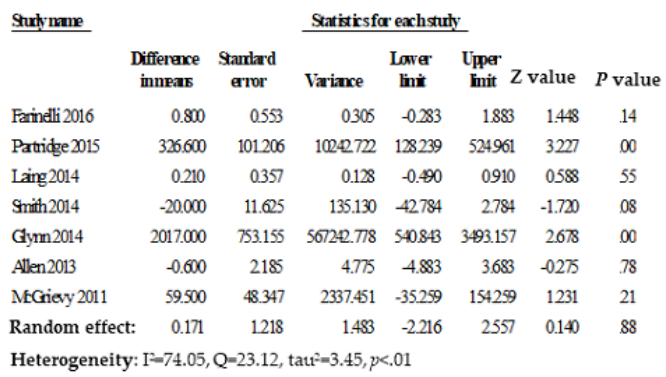

\section{Sensitivity Analysis}

Five studies evaluated the effectiveness of mobile apps used $\leq 3$ months for weight loss. Pooled findings indicated no significant difference in weight between the mobile app intervention group and the control group $(-0.004 \mathrm{~kg}, 95 \% \mathrm{CI}-0.79$ to $0.80, P=.99)$. Six studies evaluated the effectiveness of mobile apps used $>3$ months for weight loss. Pooled findings showed a significant difference in weight between the mobile app intervention group and the control group $(-1.63 \mathrm{~kg}, 95 \% \mathrm{CI}-2.64$ to -0.61 , $P<.002$ ). In addition, the pooled findings from only the randomized controlled trials showed a significant difference in

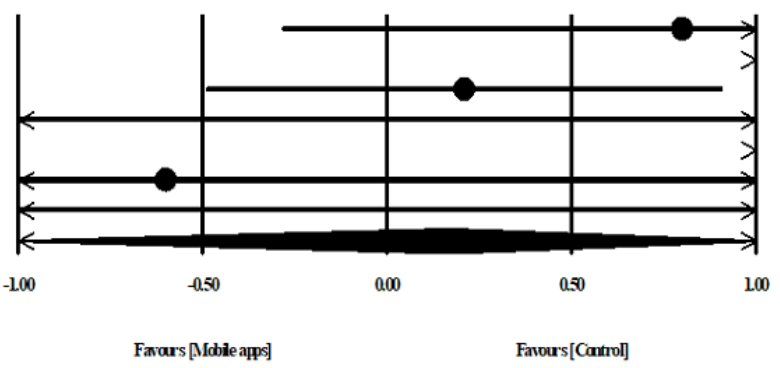

weight between the mobile app intervention group and the control group $(-1.03 \mathrm{~kg}, 95 \% \mathrm{CI}-2.05$ to $-0.025, P=.04)$ (Multimedia Appendix 3, Multimedia Appendix 4, and Multimedia Appendix 5).

\section{Publication Bias}

The meta-analysis of the observational studies had some sort of publication bias. Egger regression test was used to calculate the publication bias, and a funnel plot was drawn to visualize it. The funnel plot in Figure 6 shows no relevant publication bias.

Figure 6. Funnel plot.

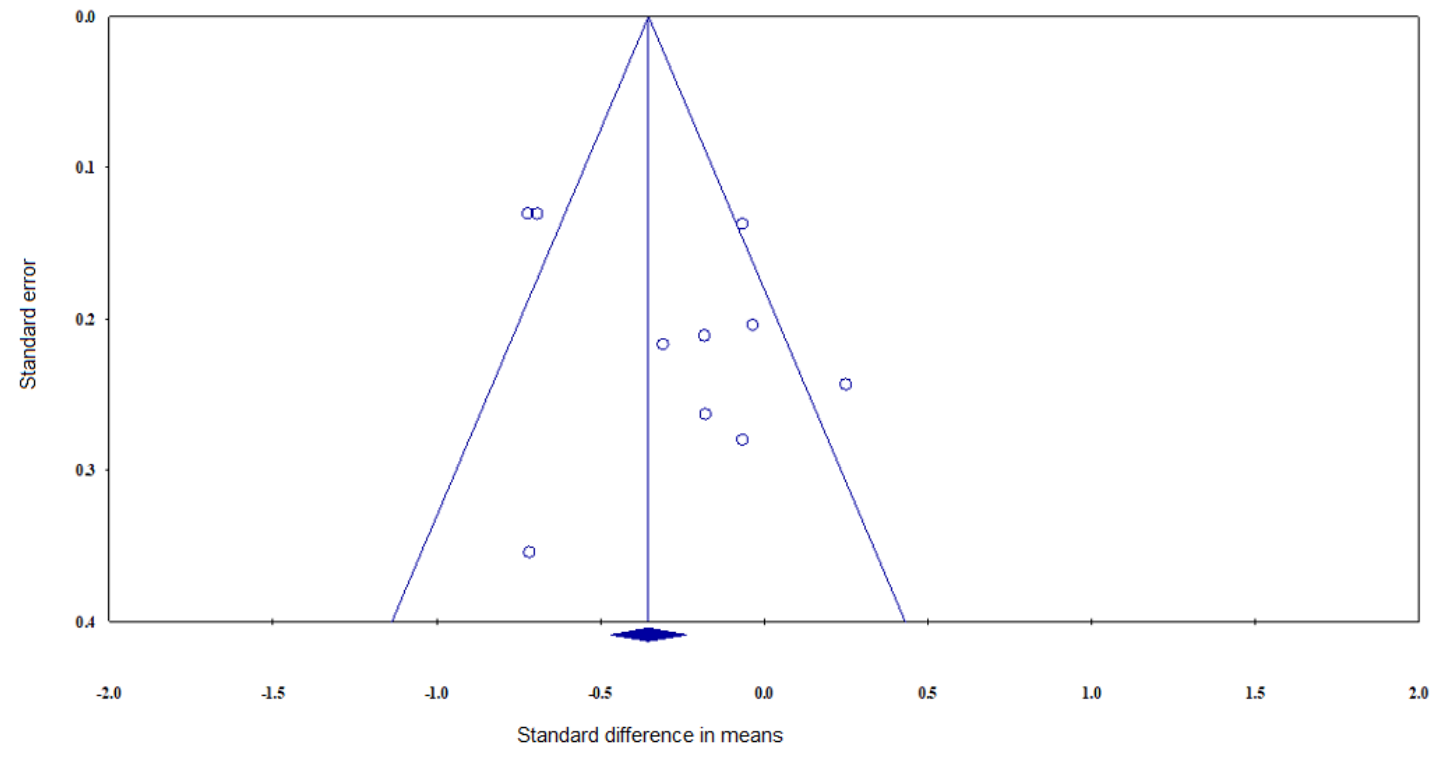




\section{Discussion}

\section{Principal Findings}

This meta-analysis evaluated the effectiveness of mobile app interventions for weight management. The meta-analysis showed a small but significant reduction in body weight $(-1.07 \mathrm{~kg})$ and a reduction in BMI $\left(-0.45 \mathrm{~kg} / \mathrm{m}^{2}\right)$. Our findings are more comprehensive across sensitivity analyses. Moreover, our findings showed that mobile app interventions promote additional positive health benefits through the maintenance of BMI and increased physical activity from baseline. The ubiquitous use of a mobile app intervention in any age group may therefore have great clinical value when compared with traditional interventions. A previous systematic review and meta-analysis reported pooled effects of app interventions compared with controls of $-1.04 \mathrm{~kg}$ for weight loss and -0.45 $\mathrm{kg} / \mathrm{m}^{2}$ for BMI [14]. The magnitude of the mobile app intervention effect in our updated meta-analysis suggests that the use of mobile app interventions is effective for promoting body weight management following an initial weight loss when compared with other interventions.

\section{Public Health Implications}

Rising obesity and physical inactivity are associated with chronic diseases and increased health costs [31]. To reduce the health care burden, researchers have already pointed out the importance of effective health communication. Digital technology provides fast and interactive communication that is easy to use and cost-effective. Mobile phones have emerged as a potential medium for interventions to assist people in maintaining health, and they have shown promising results for weight loss and increased physical activity [32]. However, a mobile app can be tailored to the individual, and information could be delivered in a more effective way that may be more realistic and feasible than conventional ways to deliver information. Several studies have investigated the efficacy of weight management using text messaging interventions. A previous meta-analysis that included 14 studies with an intervention period ranging from 1 month to 2 years found that text messaging interventions can promote weight loss $(-2.56$ $\mathrm{kg}, 95 \% \mathrm{CI}-3.46$ to -1.65$)$ [33].

Several studies have previously highlighted targeting behavioral change techniques that include dietary self-monitoring and reporting, behavior reinforcement through motivational messages, social support, setting and evaluating various goals, and setting reminders, and they are all key components to reduce and maintain weight successfully [34]. The use of mobile apps helps to improve self-awareness, provides valuable information, and can be an early indicator of health-related issues; this information and support can help to spur positive behavioral changes [35]. Results from this study suggest that even in the short term ( $<6$ months), mobile app interventions provide a generally positive effect for reducing weight and maintaining BMI. In this meta-analysis, mobile apps were related to weight loss goals, calorie goals, self-monitoring of body weight, dietary intake, real-time feedback, educational content, behavioral change plans, nutrition, physical activity, several types of trainings, and reminders of goals. Moreover, the intervention types included apps, email, internet systems, online weight trackers, physical activity planners, blog facilities for communication, internet forums, parental newsletters, seminars, spot sessions, podcasts, twitter, and printable eating charts, which were all deemed to be effective for reducing weight and maintaining BMI.

\section{Limitations}

This meta-analysis shows that a mobile app intervention has a variety of uses in reducing weight and maintaining BMI with immense benefits. However, there are several limitations in this study that we need to address. First, the findings of this study should be interpreted with caution considering the sample sizes of the included studies and considering the studies that reported on short-term periods. To increase the generalizability of our findings, a larger sample size with a longer follow-up period (at least 1 year) in diverse racial or ethnic settings is needed. Second, although heterogeneity among the studies was not high, the results were nevertheless based on a relatively small number of studies. A small number of studies also prevented us from conducting a meta-regression analysis to evaluate other factors for reducing weight and maintaining BMI.

\section{Conclusions}

The findings of this study suggest that mobile app interventions appear to be feasible and acceptable for reducing weight, maintaining BMI, and increasing physical activity, although the overall effects might be relatively modest. However, the course averages hide some variation; therefore, such interventions are highly successful in some people and completely ineffective in some people. Public awareness of safety and the benefits of weight management and physical activity should be promoted, and more studies with a larger sample and longer follow-up are needed to evaluate the potential role of a mobile phone app intervention.

\section{Acknowledgments}

We would like to thank our colleague, who is a Native English speaker, for editing our manuscript. This research was funded in part by the Ministry of Education (MOE) under grant MOE 108-6604-001-400 and DP2-109-21121-01-A-01 and the Ministry of Science and Technology (MOST) under grant MOST 108-2823-8-038-002- and 109-2222-E-038-002-MY2.

\section{Authors' Contributions}

MMI contributed to the study design and acquisition and analysis of data, as well as led the writing of the manuscript. TNP was involved in the conceptualization of the study. MMI and TNP independently screened the literature, and YCL resolved discrepancies. 
MMI, TNP, BAW, and YCL reviewed the manuscript and provided relevant feedback on the manuscript. All authors have read and approved the final version.

\section{Conflicts of Interest}

None declared.

\section{Multimedia Appendix 1}

PRISMA checklist.

[DOCX File, 17 KB-Multimedia Appendix 1]

\section{Multimedia Appendix 2}

Search words.

[DOCX File, 153 KB-Multimedia Appendix 2]

\section{Multimedia Appendix 3}

Intervention duration $\leq 3$ months.

[DOCX File, 19 KB-Multimedia Appendix 3]

\section{Multimedia Appendix 4}

Intervention duration $>3$ months.

[DOCX File , $81 \mathrm{~KB}$-Multimedia Appendix 4]

\section{Multimedia Appendix 5}

Only randomized controlled trials.

[DOCX File , $21 \mathrm{~KB}-$ Multimedia Appendix 5]

\section{References}

1. Swinburn BA, Sacks G, Hall KD, McPherson K, Finegood DT, Moodie ML, et al. The global obesity pandemic: shaped by global drivers and local environments. Lancet 2011 Aug 27;378(9793):804-814. [doi: 10.1016/S0140-6736(11)60813-1] [Medline: 21872749]

2. Chooi YC, Ding C, Magkos F. The epidemiology of obesity. Metabolism 2019 Mar;92:6-10. [doi: 10.1016/j.metabol.2018.09.005] [Medline: 30253139]

3. MacLean PS, Wing RR, Davidson T, Epstein L, Goodpaster B, Hall KD, et al. NIH working group report: Innovative research to improve maintenance of weight loss. Obesity (Silver Spring) 2015 Jan;23(1):7-15 [FREE Full text] [doi: 10.1002/oby.20967] [Medline: 25469998]

4. Franz MJ, Boucher JL, Rutten-Ramos S, VanWormer JJ. Lifestyle weight-loss intervention outcomes in overweight and obese adults with type 2 diabetes: a systematic review and meta-analysis of randomized clinical trials. J Acad Nutr Diet 2015 Sep;115(9):1447-1463. [doi: 10.1016/j.jand.2015.02.031] [Medline: 25935570]

5. Dombrowski SU, Knittle K, Avenell A, Araújo-Soares V, Sniehotta FF. Long term maintenance of weight loss with non-surgical interventions in obese adults: systematic review and meta-analyses of randomised controlled trials. BMJ 2014 May 14;348:g2646 [FREE Full text] [doi: 10.1136/bmj.g2646] [Medline: 25134100]

6. Ogilvie D, Foster CE, Rothnie H, Cavill N, Hamilton V, Fitzsimons CF, Scottish Physical Activity Research Collaboration. Interventions to promote walking: systematic review. BMJ 2007 Jun 09;334(7605):1204 [FREE Full text] [doi: 10.1136/bmj.39198.722720.BE] [Medline: 17540909]

7. Armit CM, Brown WJ, Marshall AL, Ritchie CB, Trost SG, Green A, et al. Randomized trial of three strategies to promote physical activity in general practice. Prev Med 2009 Feb;48(2):156-163. [doi: 10.1016/j.ypmed.2008.11.009] [Medline: 19100282]

8. Vodopivec-Jamsek V, de Jongh T, Gurol-Urganci I, Atun R, Car J. Mobile phone messaging for preventive health care. Cochrane Database Syst Rev 2012 Dec 12;12:CD007457 [FREE Full text] [doi: 10.1002/14651858.CD007457.pub2] [Medline: 23235643]

9. Sanou B. International Telecommunications Union Geneva. ICT Facts and Figures 2016 URL: https://www.itu.int/en/ ITU-D/Statistics/Documents/facts/ICTFactsFigures2017.pdf [accessed 2017-01-01]

10. Scott-Sheldon LA, Lantini R, Jennings EG, Thind H, Rosen RK, Salmoirago-Blotcher E, et al. Text Messaging-Based Interventions for Smoking Cessation: A Systematic Review and Meta-Analysis. JMIR Mhealth Uhealth 2016 May 20;4(2):e49 [FREE Full text] [doi: 10.2196/mhealth.5436] [Medline: 27207211] 
11. Krishna S, Boren SA, Balas EA. Healthcare via cell phones: a systematic review. Telemed J E Health 2009 Apr;15(3):231-240. [doi: 10.1089/tmj.2008.0099] [Medline: 19382860$]$

12. Ricci-Cabello I, Bobrow K, Islam SM, Chow CK, Maddison R, Whittaker R, et al. Examining Development Processes for Text Messaging Interventions to Prevent Cardiovascular Disease: Systematic Literature Review. JMIR Mhealth Uhealth 2019 Mar 29;7(3):e12191 [FREE Full text] [doi: 10.2196/12191] [Medline: 30924790]

13. Turner-McGrievy G, Tate D. Tweets, Apps, and Pods: Results of the 6-month Mobile Pounds Off Digitally (Mobile POD) randomized weight-loss intervention among adults. J Med Internet Res 2011 Dec 20;13(4):e120 [FREE Full text] [doi: 10.2196/jmir.1841] [Medline: 22186428]

14. Flores Mateo G, Granado-Font E, Ferré-Grau C, Montaña-Carreras X. Mobile Phone Apps to Promote Weight Loss and Increase Physical Activity: A Systematic Review and Meta-Analysis. J Med Internet Res 2015 Nov 10;17(11):e253 [FREE Full text] [doi: 10.2196/jmir.4836] [Medline: 26554314]

15. Stroup DF, Berlin JA, Morton SC, Olkin I, Williamson GD, Rennie D, et al. Meta-analysis of observational studies in epidemiology: a proposal for reporting. Meta-analysis Of Observational Studies in Epidemiology (MOOSE) group. JAMA 2000 Apr 19;283(15):2008-2012. [doi: 10.1001/jama.283.15.2008] [Medline: 10789670]

16. Moher D, Liberati A, Tetzlaff J, Altman DG, PRISMA Group. Preferred reporting items for systematic reviews and meta-analyses: the PRISMA statement. Ann Intern Med 2009 Aug 18;151(4):264-9, W64. [doi:

10.7326/0003-4819-151-4-200908180-00135] [Medline: 19622511]

17. Higgins JP, Thomas J, Chandler J, Cumpston M, Li T, Page MJ, et al. Cochrane. Cochrane Handbook for Systematic Reviews of Interventions Version 5 URL: https://training.cochrane.org/handbook [accessed 2015-03-25]

18. Higgins JP, Thompson SG, Deeks JJ, Altman DG. Measuring inconsistency in meta-analyses. BMJ 2003 Sep 06;327(7414):557-560 [FREE Full text] [doi: 10.1136/bmj.327.7414.557] [Medline: 12958120]

19. Patsopoulos NA, Evangelou E, Ioannidis JP. Sensitivity of between-study heterogeneity in meta-analysis: proposed metrics and empirical evaluation. Int J Epidemiol 2008 Oct;37(5):1148-1157 [FREE Full text] [doi: 10.1093/ije/dyn065] [Medline: $\underline{18424475]}$

20. Allman-Farinelli M, Partridge SR, McGeechan K, Balestracci K, Hebden L, Wong A, et al. A Mobile Health Lifestyle Program for Prevention of Weight Gain in Young Adults (TXT2BFiT): Nine-Month Outcomes of a Randomized Controlled Trial. JMIR Mhealth Uhealth 2016 Jun 22;4(2):e78 [FREE Full text] [doi: 10.2196/mhealth.5768] [Medline: 27335237]

21. Patel ML, Hopkins CM, Brooks TL, Bennett GG. Comparing Self-Monitoring Strategies for Weight Loss in a Smartphone App: Randomized Controlled Trial. JMIR Mhealth Uhealth 2019 Feb 28;7(2):e12209 [FREE Full text] [doi: 10.2196/12209] [Medline: $\underline{30816851]}$

22. Lee W, Chae YM, Kim S, Ho SH, Choi I. Evaluation of a mobile phone-based diet game for weight control. J Telemed Telecare 2010;16(5):270-275. [doi: 10.1258/jtt.2010.090913] [Medline: 20558620]

23. Carter MC, Burley VJ, Nykjaer C, Cade JE. Adherence to a smartphone application for weight loss compared to website and paper diary: pilot randomized controlled trial. J Med Internet Res 2013 Apr 15;15(4):e32 [FREE Full text] [doi: 10.2196/jmir.2283] [Medline: 23587561]

24. Allen JK, Stephens J, Dennison Himmelfarb CR, Stewart KJ, Hauck S. Randomized controlled pilot study testing use of smartphone technology for obesity treatment. J Obes 2013;2013:151597 [FREE Full text] [doi: 10.1155/2013/151597] [Medline: 24392223]

25. Brindal E, Hendrie G, Freyne J, Coombe M, Berkovsky S, Noakes M. Design and pilot results of a mobile phone weight-loss application for women starting a meal replacement programme. J Telemed Telecare 2013 Apr;19(3):166-174. [doi: 10.1177/1357633X13479702] [Medline: 23520213]

26. Laing BY, Mangione CM, Tseng C, Leng M, Vaisberg E, Mahida M, et al. Effectiveness of a smartphone application for weight loss compared with usual care in overweight primary care patients: a randomized, controlled trial. Ann Intern Med 2014 Nov 18;161(10 Suppl):S5-12 [FREE Full text] [doi: 10.7326/M13-3005] [Medline: 25402403]

27. Glynn LG, Hayes PS, Casey M, Glynn F, Alvarez-Iglesias A, Newell J, et al. Effectiveness of a smartphone application to promote physical activity in primary care: the SMART MOVE randomised controlled trial. Br J Gen Pract 2014 Jul;64(624):e384-e391 [FREE Full text] [doi: 10.3399/bjgp14X680461] [Medline: 24982490]

28. Smith JJ, Morgan PJ, Plotnikoff RC, Dally KA, Salmon J, Okely AD, et al. Smart-phone obesity prevention trial for adolescent boys in low-income communities: the ATLAS RCT. Pediatrics 2014 Sep;134(3):e723-e731. [doi: 10.1542/peds.2014-1012] [Medline: 25157000]

29. Hebden L, Cook A, van der Ploeg HP, King L, Bauman A, Allman-Farinelli M. A mobile health intervention for weight management among young adults: a pilot randomised controlled trial. J Hum Nutr Diet 2014 Aug;27(4):322-332. [doi: 10.1111/jhn.12155] [Medline: 23992038]

30. Partridge SR, McGeechan K, Hebden L, Balestracci K, Wong AT, Denney-Wilson E, et al. Effectiveness of a mHealth Lifestyle Program With Telephone Support (TXT2BFiT) to Prevent Unhealthy Weight Gain in Young Adults: Randomized Controlled Trial. JMIR Mhealth Uhealth 2015 Jun 15;3(2):e66 [FREE Full text] [doi: 10.2196/mhealth.4530] [Medline: 26076688] 
31. Holmes WS, Moorhead SA, Coates VE, Bond RR, Zheng H. Impact of digital technologies for communicating messages on weight loss maintenance: a systematic literature review. Eur J Public Health 2019 Apr 01;29(2):320-328. [doi: 10.1093/eurpub/cky171] [Medline: 30239699 ]

32. Shaw R, Bosworth H. Short message service (SMS) text messaging as an intervention medium for weight loss: A literature review. Health Informatics J 2012 Dec;18(4):235-250 [FREE Full text] [doi: 10.1177/1460458212442422] [Medline: 23257055]

33. Siopis G, Chey T, Allman-Farinelli M. A systematic review and meta-analysis of interventions for weight management using text messaging. J Hum Nutr Diet 2015 Feb;28 Suppl 2:1-15. [doi: 10.1111/jhn.12207] [Medline: 24480032]

34. Greaves CJ, Sheppard KE, Abraham C, Hardeman W, Roden M, Evans PH, IMAGE Study Group. Systematic review of reviews of intervention components associated with increased effectiveness in dietary and physical activity interventions. BMC Public Health 2011 Feb 18;11:119 [FREE Full text] [doi: 10.1186/1471-2458-11-119] [Medline: 21333011]

35. Liu F, Kong X, Cao J, Chen S, Li C, Huang J, et al. Mobile phone intervention and weight loss among overweight and obese adults: a meta-analysis of randomized controlled trials. Am J Epidemiol 2015 Mar 01;181(5):337-348 [FREE Full text] [doi: 10.1093/aje/kwu260] [Medline: 25673817]

Edited by G Eysenbach; submitted 12.11.19; peer-reviewed by E Bellei, LK Chen, A Griffin, C Carrion, K Tamura; comments to author 08.03.20; revised version received 16.03.20; accepted 07.04.20; published 22.07.20

Please cite as:

Islam MM, Poly TN, Walther BA, (Jack) Li YC

Use of Mobile Phone App Interventions to Promote Weight Loss: Meta-Analysis

JMIR Mhealth Uhealth 2020;8(7):e17039

URL: https://mhealth.jmir.org/2020/7/e17039

doi: 10.2196/17039

PMID: 32706724

CMd.Mohaimenul Islam, Tahmina Nasrin Poly, Bruno Andres Walther, Yu-Chuan (Jack) Li. Originally published in JMIR mHealth and uHealth (http://mhealth.jmir.org), 22.07.2020. This is an open-access article distributed under the terms of the Creative Commons Attribution License (https://creativecommons.org/licenses/by/4.0/), which permits unrestricted use, distribution, and reproduction in any medium, provided the original work, first published in JMIR mHealth and uHealth, is properly cited. The complete bibliographic information, a link to the original publication on http://mhealth.jmir.org/, as well as this copyright and license information must be included. 